\title{
On $n$-phantom and $n$-Ext-phantom Morphisms
}

\author{
Kaiyang Lan and Bo Lu*
}

\begin{abstract}
This paper extends many conclusions based on phantom envelopes and Ext-phantom covers of modules, and we find that many important properties still hold after replacing phantom and Ext-phantom with $n$-phantom and $n$-Ext-phantom respectively. In addition, we also obtain some extra results. Specifically, we give a characterization of the weak dimensions of rings in terms of $n$-phantom envelopes and $n$-Ext-phantom covers of modules with the unique mapping property respectively. We show that $\mathrm{wD}(R) \leq 2 n$ whenever every right $R$-module has an $n$-phantom envelope with the unique mapping property or every left $R$-module has an $n$-Ext-phantom cover with the unique mapping property over left coherent rings.
\end{abstract}

\section{Introduction}

Unless stated otherwise, throughout this paper, all rings are associative with identity 1 and all modules are unitary, that is, for all $x \in M$, we have $1 x=x$, where $M$ is a left $R$ module. For a ring $R,{ }_{R} M\left(M_{R}\right)$ denotes a left (right) $R$-module. The character module $\operatorname{Hom}_{Z}(M, Q / Z)$ of $M$ is denoted by $M^{+}$and $\delta_{M}: M \rightarrow M^{++}$stands for the canonical embedding. $\mathrm{fd}(M)$ is the flat dimension of $M$ and $\mathrm{wD}(R)$ stands for the weak global dimension of $R \cdot \operatorname{Hom}(M, N)$ and $M \otimes N$ mean $\operatorname{Hom}_{R}(M, N)$ and $M \otimes_{R} N$, respectively. And in similar way, we use $\operatorname{Ext}^{n}(M, N)$ and $\operatorname{Tor}_{n}(M, N)$ to stand for the derived functors $\operatorname{Ext}_{R}^{n}(M, N)$ and $\operatorname{Tor}_{n}^{R}(M, N)$, respectively. Let $R$-Mor denote the category whose objects are left $R$-module morphisms, and where the morphism from a left $R$-module morphism $M_{1} \stackrel{\alpha}{\rightarrow} M_{2}$ to a left $R$-module morphism $N_{1} \stackrel{\beta}{\rightarrow} N_{2}$ is a pair of left $R$-module morphisms $\left(M_{1} \stackrel{\sigma}{\rightarrow} N_{1}, M_{2} \stackrel{\tau}{\rightarrow} N_{2}\right)$ such that the following diagram

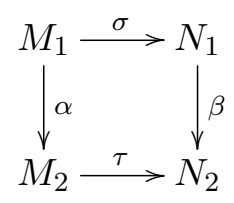

is commutative.

Received August 11, 2020; Accepted March 21, 2021.

Communicated by Kunio Yamagata.

2020 Mathematics Subject Classification. 16D90, 16E30, 18G25.

Key words and phrases. $n$-phantom morphism, n-Ext-phantom morphism, (pre)cover, (pre)envelope.

*Corresponding author. 
Let $R$ be a ring, we use $R$-Mod (respectively, $\operatorname{Mod}-R$ ) to represent the category of left (respectively, right) $R$-modules and $\mathrm{Ab}$ the category of abelian groups. We know that an additive subbifunctor of the bifunctor $\operatorname{Hom}_{R}(-,-): R$ - $\operatorname{Mod}^{\mathrm{op}} \times R$ - Mod $\rightarrow \mathrm{Ab}$ is called an ideal $\mathcal{I}$ of $R$-Mod. That is, given left $R$-morphisms $f, g, h, t$ with $f, g \in \mathcal{I}$, we have $f+g \in \mathcal{I}$ and $h f t \in \mathcal{I}$ provided they are defined. The ideal of phantom morphisms is an important instance of ideals. The exploration of phantom morphisms has its roots in topology in the searching of maps between CW complexes [18 and was first introduced into the field of a triangulated category by Neeman in [20]. In 2002, the theory of phantom morphisms was also developed in the stable category of a finite group ring by Benson in [1]. Soon after, the definition of a phantom morphism was generalized to the category of modules over any ring by Herzog in [8]. He called a morphism $f: M \rightarrow N$ in $R$-Mod a phantom morphism if the induced morphism

$$
\operatorname{Tor}_{1}(A, f): \operatorname{Tor}_{1}(A, M) \rightarrow \operatorname{Tor}_{1}(A, N)
$$

of abelian groups is zero for any (finitely presented) right $R$-module $A$. Similarly, a morphism $g: X \rightarrow Y$ in $R$-Mod is called an Ext-phantom morphism [9] if the induced morphism

$$
\operatorname{Ext}^{1}(B, g): \operatorname{Ext}^{1}(B, X) \rightarrow \operatorname{Ext}^{1}(B, Y)
$$

of abelian groups is zero for any finitely presented left $R$-module $B$. Ext-phantom morphisms are also called cophantom morphisms in the work of $\mathrm{Fu}$ et al. in 6]. In 2018, Mao introduced the concept of $n$-phantom morphism [14], which is a higher dimensional generalization of phantom morphisms.

We know that the concepts of preenvelopes and precovers (approximations) were introduced by Enochs in the eighties of the twentieth century [4]. Let $\mathcal{A}$ be any category and $\mathcal{C}$ a class of objects in $\mathcal{A}$. We know that a morphism $\varphi: X \rightarrow Y$ in $\mathcal{A}$ is a $\mathcal{C}$-preenvelope of $X[4]$ if $Y \in \mathcal{C}$ and, for any morphism $f: X \rightarrow Z$ with $Z \in \mathcal{C}$, there is a morphism $g: Y \rightarrow Z$ such that $g \varphi=f$. A $\mathcal{C}$-preenvelope $\varphi: X \rightarrow Y$ is said to be a $\mathcal{C}$-envelope of $X$ if every endomorphism $g: Y \rightarrow Y$ such that $g \varphi=\varphi$ is an isomorphism. Dually, we have the definitions of a $\mathcal{C}$-precover and a $\mathcal{C}$-cover. Covers and envelopes provide common frames for a lot of classical notions such as projective covers and injective envelopes, and the results is extremely fruitful for general module theory as well as for representation theory. Recently, ideal approximation theory has been raised and developed by $\mathrm{Fu}$ et al. in [6,7]. Let $\mathcal{I}$ be an ideal of $R$-Mod. A morphism $\varphi: M \rightarrow N$ in $\mathcal{I}$ is an $\mathcal{I}$-preenvelope of $M[6$ if for any morphism $\psi: M \rightarrow C$ in $\mathcal{I}$, there is a morphism $\theta: N \rightarrow C$ such that $\theta \varphi=\psi$. An $\mathcal{I}$-preenvelope $\varphi: M \rightarrow N$ is called an $\mathcal{I}$-envelope if every endomorphism $h$ of $N$ such that $h \varphi=\varphi$ is an isomorphism. An $\mathcal{I}$-precover and an $\mathcal{I}$-cover of a module are defined dually. It is easy to know that ideal approximation theory is a generalization of 
the classical theory of envelopes and covers (approximation theory) since it just needs to be set forth by the morphisms instead of the objects. In recent years, ideal approximation theory has experienced an ever-increasing development 6, 7].

In 2007, Herzog proved that any left $R$-module has a phantom cover which is an epimorphism with its kernel pure injective. In 2013, Mao proved that any left $R$-module has an Ext-phantom preenvelope which is a monomorphism with its cokernel pure projective [13]. In 2018, Mao proved that every left $R$-module has an $n$-phantom cover in $R$-Mod [14. We note that the existence of Ext-phantom preenvelopes is a special case of the works [6. Theorems 32 and 33]. In Section 2 of this paper, we prove that if a monomorphism is an $n$-phantom envelope of a left $R$-module, then its cokernel is pure projective; if $R$ is a left coherent ring and an epimorphism is an $n$-Ext-phantom cover of a left $R$-module, then its kernel is pure injective. In addition, We describe $n$-phantom envelopes and $n$-Ext-phantom covers of modules using left $F C$ rings and left coherent rings.

In 2016, Mao investigated when every module has a phantom preenvelope or an Extphantom cover. And in 2018, under certain conditions, he proved that every module has an $n$-phantom preenvelope or an $n$-Ext-phantom cover [14]. In Section 3 of this paper, we study every module has an $n$-phantom envelope or an $n$-Ext-phantom cover under certain conditions. In addition, we describe the weak dimension of a ring $R$ according to $n$-phantom envelopes and $n$-Ext-phantom covers with some special conditions such as coherent rings or having unique mapping properties. We conclude this section by giving the following two definitions.

Definition 1.1. 21, Page 461] Let $R$ be a ring and $M$ a left $R$-module. If there is a finite flat resolution

$$
0 \longrightarrow F_{n} \longrightarrow \cdots \longrightarrow F_{1} \longrightarrow F_{0} \longrightarrow M \longrightarrow 0
$$

then $\operatorname{fd}(M) \leq n$. If no such finite resolution exists, then we write $\operatorname{fd}(M)=\infty$; otherwise, $\operatorname{fd}(M)=n$ if $n$ is the length of the shortest flat resolution of $M$.

Definition 1.2. [21, Page 474] Let $R$ be a ring. The weak dimension of $R$ is defined by

$$
\mathrm{wD}(R)=\sup \{\operatorname{fd}(M) \mid M \in R-\operatorname{Mod}\} .
$$

2. $n$-phantom and n-Ext-phantom morphisms

We start by the following definitions.

Recall that a morphism $f: M \rightarrow N$ in $R$-Mod is an $n$-phantom morphism [14] if the induced morphism

$$
\operatorname{Tor}_{n}(A, f): \operatorname{Tor}_{n}(A, M) \rightarrow \operatorname{Tor}_{n}(A, N)
$$


of abelian groups is zero for any (finitely presented) right $R$-module $A$. Analogously, a morphism $g: X \rightarrow Y$ in $R$-Mod is called an $n$-Ext-phantom morphism if the induced morphism

$$
\operatorname{Ext}^{n}(B, g): \operatorname{Ext}^{n}(B, X) \rightarrow \operatorname{Ext}^{n}(B, Y)
$$

of abelian groups is zero for any finitely presented left $R$-module $B$.

Obviously, 1-phantom morphism in $R$-Mod is exactly a phantom morphism; 1-Extphantom morphism in $R$-Mod is exactly an Ext-phantom morphism. And both $n$-phantom and $n$-Ext-phantom morphisms in $R$-Mod are ideals in $R$-Mod.

We also recall that an exact sequence $0 \rightarrow A \rightarrow B \rightarrow C \rightarrow 0$ of left $R$-modules is pure 23 if the induced sequence $\operatorname{Hom}(X, B) \rightarrow \operatorname{Hom}(X, C) \rightarrow 0$ is exact for any finitely presented left $R$-module $X$, or equivalently, if the induced sequence $0 \rightarrow Y \otimes A \rightarrow Y \otimes B$ is exact for any (finitely presented) right $R$-module $Z$ by [23, Proposition 3]. In this case, $A \rightarrow B$ is called a pure monomorphism and $B \rightarrow C$ is called a pure epimorphism. A left $R$-module $G$ is called pure injective (respectively, pure projective) if the functor $\operatorname{Hom}(-, G)$ (respectively, $\operatorname{Hom}(G,-)$ ) preserves the exactness of any pure exact sequence of left $R$-modules. And we also know that a $\operatorname{ring} R$ is left coherent 11] if every finitely generated left ideal of $R$ is finitely presented.

The next lemma comes from 16 and it will be used frequently.

Lemma 2.1. Let $0 \rightarrow X \stackrel{\alpha}{\rightarrow} Y \stackrel{\beta}{\rightarrow} Z \rightarrow 0$ be an exact sequence in $R$-Mod and $n$ a positive integer. Then the following conditions are equivalent:

(1) $0 \rightarrow X \stackrel{\alpha}{\rightarrow} Y \stackrel{\beta}{\rightarrow} Z \rightarrow 0$ is pure.

(2) The induced sequence

$$
0 \rightarrow \operatorname{Tor}_{n}(A, X) \rightarrow \operatorname{Tor}_{n}(A, Y) \rightarrow \operatorname{Tor}_{n}(A, Z) \rightarrow 0
$$

is exact for any right $R$-module $A$.

Furthermore, if $R$ is a left coherent ring, then the above conditions are equivalent to

(3) The induced sequence

$$
0 \rightarrow \operatorname{Ext}^{n}(G, X) \rightarrow \operatorname{Ext}^{n}(G, Y) \rightarrow \operatorname{Ext}^{n}(G, Z) \rightarrow 0
$$

is exact for any finitely presented left $R$-module $G$.

Recall that a left $R$-module $X$ is FP-injective $[22]$ if $\operatorname{Ext}_{R}^{1}(B, X)=0$ for every finitely presented left $R$-module $B$. The FP-injective dimension of $X$, denoted by FP-id $(X)$, is defined to be the smallest nonnegative integer $n$ such that $\operatorname{Ext}_{R}^{n+1}(Y, X)=0$ for any finitely presented left $R$-module $Y$. If no such $n$ exists, set $\operatorname{FP}$-id $(X)=\infty$. In 16 , Corollaries 2.2 
and 2.3], the author proved that a left $R$-module $M$ is flat if and only if every pure monomorphism $M \rightarrow N$ is a phantom morphism. In addition, if $R$ is a left coherent ring, he also proved that a left $R$-module $N$ is FP-injective if and only if every pure epimorphism $M \rightarrow N$ is an Ext-phantom morphism. Here we have the following two results.

Proposition 2.2. Let $R$ be a ring and $n$ a positive integer. Then the following conditions are equivalent for a left $R$-module $X$ :

(1) $\operatorname{fd}(X) \leq n-1$

(2) Every monomorphism $X \rightarrow N$ is an $n$-phantom morphism for any left $R$-module $N$.

(3) Every pure monomorphism $X \rightarrow N$ is an $n$-phantom morphism for any left $R$-module $N$.

(4) There exists an $n$-phantom and pure monomorphism $f: X \rightarrow N$ for any left $R$ module $N$.

Proof. $(1) \Rightarrow(2)$ is trivial since $\mathrm{fd}(X) \leq n-1$ if and only if $\operatorname{Tor}_{n}(B, X)=0$ for all right $R$-module $B$.

$(2) \Rightarrow(3)$ is clear.

$(3) \Rightarrow(4)$ is easy since $X$ is a pure submodule of a pure injective left $R$-module by 23 , Corollary 6].

$(4) \Rightarrow(1)$. For any right $R$-module $Y$, we have

$$
\operatorname{Tor}_{n}(Y, f): \operatorname{Tor}_{n}(Y, X) \rightarrow \operatorname{Tor}_{n}(Y, N)
$$

is a monomorphism by Lemma 2.1. Since $\operatorname{Tor}_{n}(Y, f)=0, \operatorname{Tor}_{n}(Y, X)=0$. So $\mathrm{fd}(X) \leq$ $n-1$.

Proposition 2.3. Let $R$ be a ring and $n$ a positive integer. Then the following conditions are equivalent for a left $R$-module $Y$ :

(1) $\operatorname{FP}-i d(Y) \leq n-1$.

(2) Every epimorphism $M \rightarrow Y$ is an $n$-Ext-phantom morphism for any left $R$-module $M$.

(3) Every pure epimorphism $M \rightarrow Y$ is an $n$-Ext-phantom morphism for any left $R$ module $M$.

(4) There exist an $n$-Ext-phantom and pure epimorphism $g: M \rightarrow Y$ for any left $R$ module $M$. 
Proof. $(1) \Rightarrow(2)$ is trivial since $\operatorname{FP}-\mathrm{id}(Y) \leq n-1$ if and only if $\operatorname{Ext}_{R}^{n}(F, Y)=0$ for all finitely presented right $R$-modules $F$.

$(2) \Rightarrow(3)$ is clear.

$(3) \Rightarrow(4)$ is easy since $Y$ is a pure quotient of a pure projective left $R$-module by 23 , Proposition 1].

$(4) \Rightarrow(1)$. For any finitely presented left $R$-module $B$, we have

$$
\operatorname{Ext}^{n}(B, g): \operatorname{Ext}^{n}(B, M) \rightarrow \operatorname{Ext}^{n}(B, Y)
$$

is an epimorphism by Lemma 2.1. Since $\operatorname{Ext}^{n}(B, g)=0, \operatorname{Ext}^{n}(B, Y)=0$. So FP-id( $\left.Y\right) \leq$ $n-1$.

In [16], Mao proved that if $R$ is a left coherent ring and $f: M \rightarrow N$ is a phantom preenvelope in Mod- $R$, then $f^{+}: N^{+} \rightarrow M^{+}$is an Ext-phantom precover in $R$-Mod. By the similar method, we obtain the following results.

Proposition 2.4. Let $R$ be a left coherent ring and $n$ a positive integer. If $\alpha: A \rightarrow B$ is an $n$-phantom preenvelope in Mod- $R$, then

(1) $\alpha^{+}: B^{+} \rightarrow A^{+}$is an $n$-Ext-phantom precover of $A^{+}$in $R$-Mod.

(2) $\alpha: A \rightarrow B$ is an n-phantom envelope of $A$ if $\alpha^{+}: B^{+} \rightarrow A^{+}$is an $n$-Ext-phantom cover of $A^{+}$.

Proof. (1) By [14, Proposition 2.10], $\alpha^{+}: B^{+} \rightarrow A^{+}$is an $n$-Ext-phantom morphism in $R$-Mod. For any $n$-Ext-phantom morphism $\beta: G \rightarrow A^{+}$, we have $\beta^{+}: A^{++} \rightarrow G^{+}$is an $n$-phantom morphism in Mod- $R$ by [14, Proposition 2.10] since $R$ is left coherent. Since $\beta^{+} \delta_{A}: A \stackrel{\delta_{A}}{\rightarrow} A^{++} \stackrel{\beta^{+}}{\rightarrow} G^{+}$is an $n$-phantom morphism in Mod- $R$, there exists a morphism $h: B \rightarrow G^{+}$such that $h \alpha=\beta^{+} \delta_{A}$ since $\alpha$ is an $n$-phantom preenvelope. So $\alpha^{+} h^{+}=\delta_{A}^{+} \beta^{++}$. Then we consider the following commutative diagram

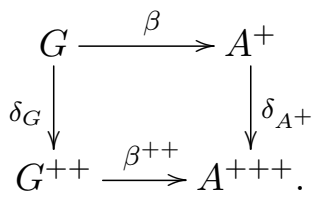

So we have

$$
\alpha^{+}\left(h^{+} \delta_{G}\right)=\delta_{A}^{+}\left(\beta^{++} \delta_{G}\right)=\left(\delta_{A}^{+} \delta_{A^{+}}\right) \beta=\beta .
$$

Hence, $\alpha^{+}: B^{+} \rightarrow A^{+}$is an $n$-Ext-phantom precover of $A^{+}$in $R$-Mod.

(2) Let $\gamma: B \rightarrow B$ satisfy that $\gamma \alpha=\alpha$. Then we have $\alpha^{+} \gamma^{+}=\alpha^{+}$. Since $\alpha^{+}: B^{+} \rightarrow$ $A^{+}$is an $n$-Ext-phantom cover, $\gamma^{+}$is an isomorphism and so $\gamma$ is an isomorphism. Thus, $\alpha: A \rightarrow B$ is an $n$-phantom envelope of $A$. 
It is well known that many kinds of rings can be characterized by the existence of flat (pre)envelopes and FP-injective covers of modules. Conversely, in this section we will investigate that what kind of rings can satisfying that every module has an $n$-phantom (pre)envelope or an $n$-Ext-phantom cover (which is a monomorphism or an epimorphism, respectively).

For the proof of the next lemma one can see [14, Corollary 2.14].

Lemma 2.5. Let $R$ be a left coherent ring and $n$ a positive integer. Then every right $R$-module has an $n$-phantom preenvelope.

Proposition 2.6. Let $R$ be a left coherent ring and $n$ a positive integer. If the class of $n$-phantom morphisms in Mor- $R$ is closed under inverse limits, then every right $R$-module has an $n$-phantom envelope.

Proof. It is easy to see that the category Mor- $R$ is equivalent to the category Mod- $T$, where $T$ is the lower triangular $2 \times 2$ matrix ring over $R$. And $T$ is a left coherent ring by 12 , Theorem 2.3]. Then by [5, Corollary 6.3.5] we have every right $R$-module morphism has an $n$-phantom envelope since the class of $n$-phantom morphisms is closed under summands. Thus, every right $R$-module has an $n$-phantom envelope by [16, Lemma 3.4].

Recall that a ring $R$ is a left $F C$ ring $[2]$ if $R$ is left coherent and ${ }_{R} R$ is FP-injective. By the works [3, Corollary 4.2] and [17, Proposition 3.7], a ring $R$ is a left $F C$ ring if and only if every right $R$-module has a flat preenvelope which is monic if and only if every left $R$-module has an FP-injective cover which is epic. Here we have the following result.

Proposition 2.7. Let $R$ be a left $F C$ ring and $n$ a positive integer.

(1) Every right $R$-module has an $n$-phantom preenvelope which is a monomorphism.

(2) Every left $R$-module has an $n$-Ext-phantom cover which is an epimorphism.

Proof. (1) Every right $R$-module $A$ has an $n$-phantom preenvelope $f: A \rightarrow B$ by Lemma 2.5 . Note that $A$ can be embedded in an injective right $R$-module and so can be embedded in a flat right $R$-module $F$ by $[10$, Theorem 3.10] since $R$ is left coherent. Let $h: A \rightarrow F$ be the canonical inclusion. Then $h$ is an $n$-phantom morphism and there exists a morphism $g: B \rightarrow F$ such that $g f=h$ since $f$ is an $n$-phantom preenvelope. Thus, $f: A \rightarrow B$ is a monomorphism since $h$ is monic.

(2) Every left $R$-module $A$ has an $n$-Ext-phantom cover $f: X \rightarrow A$ by 14 , Corollary 2.14]. There exists an epimorphism $\beta: \oplus_{R} R \rightarrow A$. Since FP-injective $R$-module is closed under direct sums and ${ }_{R} R$ is FP-injective, $\oplus_{R} R$ is FP-injective. Then $\beta$ is an $n$-Ext-phantom morphism and there exists a morphism $h: \oplus_{R} R \rightarrow X$ such that $f h=\beta$ since $f$ is an $n$-Ext-phantom cover. Thus, $f$ is an epimorphism since $\beta$ is epic. 
Now we exhibiting our main results in this article.

Let $n$ be a positive integer. By [14, Corollary 2.14], every left $R$-module has an $n$-phantom cover in $R$-Mod. If $R$ is a right coherent ring, then every left $R$-module has an $n$-phantom preenvelope in $R$-Mod. If $R$ is a left coherent ring, then every left $R$-module has an $n$-Ext-phantom cover and an $n$-Ext-phantom preenvelope in $R$-Mod. By [16, Theorems 2.4 and 2.5], if a left $R$-module $X$ has a phantom preenvelope $\alpha: X \rightarrow Y$ which is a monomorphism, then $X$ has a phantom preenvelope $\phi: X \rightarrow W$ with coker $(\phi)$ pure projective. If $R$ is a left coherent ring and a left $R$-module $M$ has an Ext-phantom precover $f: N \rightarrow M$ which is an epimorphism, then $M$ has an Ext-phantom precover $\pi: H \rightarrow M$ with $\operatorname{ker}(\pi)$ pure injective. Now according to this, we consider the cokernel of an $n$-phantom envelope and the kernel of an $n$-Ext-phantom cover.

Theorem 2.8. Let $A$ be a left $R$-module and $n$ a positive integer.

(1) If $A$ has an n-phantom preenvelope $\alpha: A \rightarrow B$ which is a monomorphism, then $A$ has an $n$-phantom preenvelope $\phi: A \rightarrow W$ with $\operatorname{coker}(\phi)$ pure projective.

(2) If $\alpha: A \rightarrow B$ is an n-phantom envelope of $A$ which is a monomorphism, then $\operatorname{coker}(\alpha)$ is pure projective.

Proof. (1) Let $G=\operatorname{coker}(\alpha)$. There exists a pure exact sequence $0 \rightarrow V \rightarrow U \stackrel{\psi}{\rightarrow} G \rightarrow 0$, where $U$ is a pure projective left $R$-module. Consider the following pullback diagram

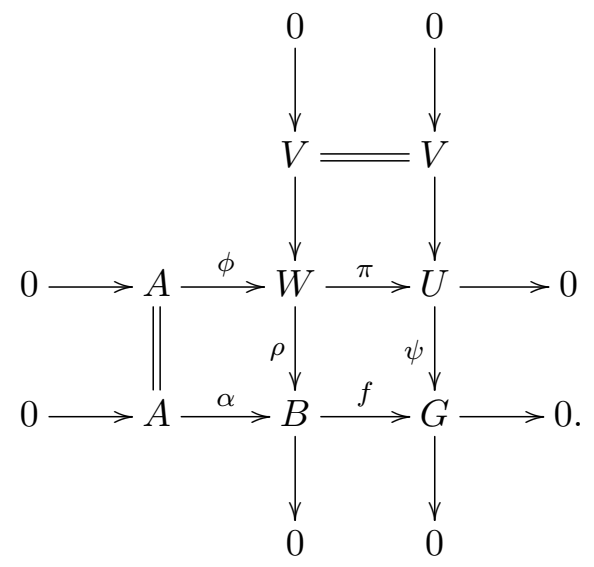

For any right $R$-module $C$, we get the following commutative diagram with exact rows:

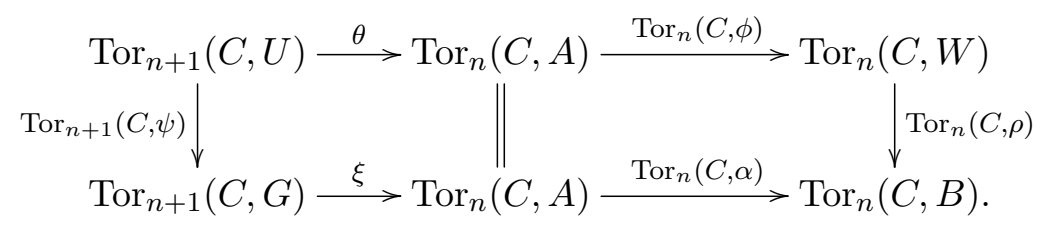

Since $\operatorname{Tor}_{n}(C, \alpha)=0, \xi$ is an epimorphism. Since $\operatorname{Tor}_{n+1}(C, \psi)$ is an epimorphism by Lemma 2.1. $\theta=\xi \operatorname{Tor}_{n+1}(C, \psi)$ is an epimorphism. So $\operatorname{Tor}_{n}(C, \phi)=0$. Thus, $\phi: A \rightarrow W$ 
is an $n$-phantom morphism. For any $n$-phantom morphism $\sigma: A \rightarrow Z$, there exists a morphism $\theta: B \rightarrow Z$ such that $\theta \alpha=\sigma$ since $\alpha$ is an $n$-phantom preenvelope. So $(\theta \rho) \phi=$ $\theta \alpha=\sigma$. Hence, $\phi: A \rightarrow W$ is an $n$-phantom preenvelope with the cokernel $U$ of $\phi$ pure projective.

(2) By (1), $A$ has an $n$-phantom preenvelope $\phi: A \rightarrow W$ which is a monomorphism with the cokernel $U$ of $\phi$ pure projective. Since $\alpha: A \rightarrow B$ is an $n$-phantom envelope, there exists a morphism $\gamma: B \rightarrow W$ such that $\phi=\gamma \alpha$. Also there exists $\omega: G \rightarrow U$ by Five Lemma such that $\pi \gamma=\omega f$. Then, we get the following commutative diagram with exact rows:

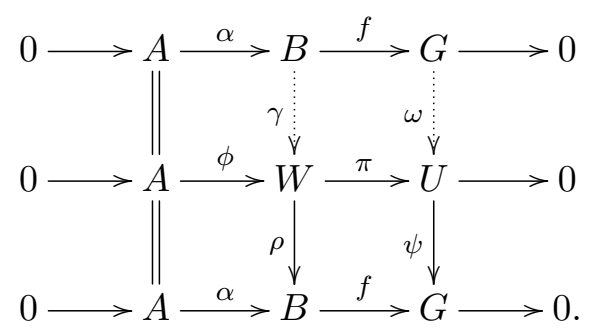

Since $\alpha$ is an $n$-phantom envelope, $\rho \gamma$ is an isomorphism. Hence, $\psi \omega$ is an isomorphism by Five Lemma again. Thus, $G$ is isomorphic to a direct summand of $U$ and so is pure projective.

Theorem 2.9. Let $R$ be a left coherent ring, $Y$ a left $R$-module and $n$ a positive integer.

(1) If $Y$ has an $n$-Ext-phantom precover $g: X \rightarrow Y$ which is an epimorphism, then $Y$ has an $n$-Ext-phantom precover $\beta: H \rightarrow Y$ with $\operatorname{ker}(\beta)$ pure injective.

(2) If $g: X \rightarrow Y$ is an $n$-Ext-phantom cover of $Y$ which is an epimorphism, then $\operatorname{ker}(g)$ is pure injective.

Proof. (1) Let $K=\operatorname{ker}(g)$. There exists a pure exact sequence $0 \rightarrow K \stackrel{\varphi}{\rightarrow} C \rightarrow L \rightarrow 0$, where $C$ is a pure injective left $R$-module. We consider the following pushout diagram

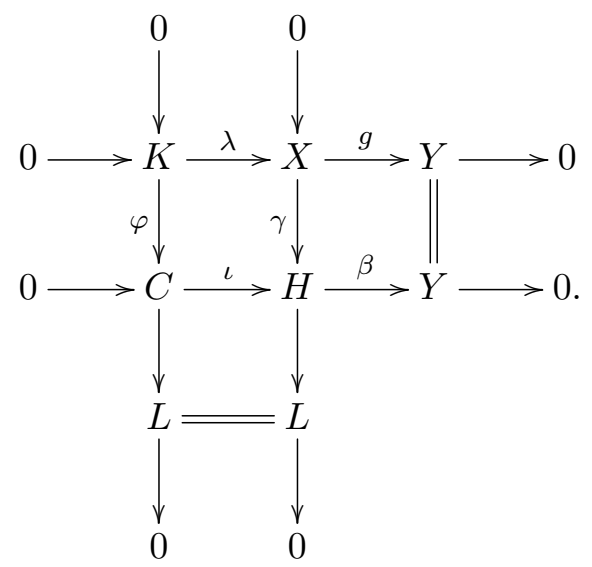


Let $B$ be a finitely presented left $R$-module. Then we get the following commutative diagram with exact rows:

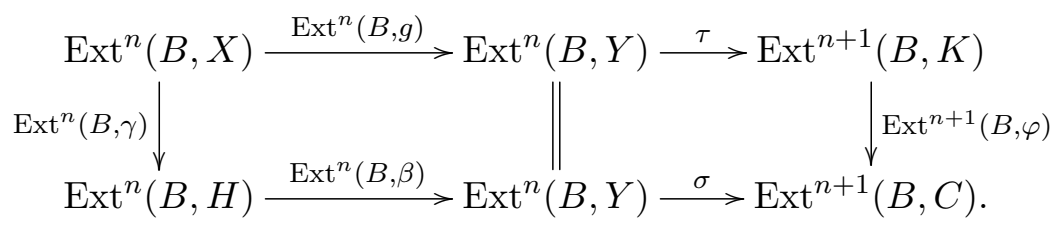

Since $\operatorname{Ext}^{n}(B, g)=0, \tau$ is a monomorphism. Also $\operatorname{Ext}^{n+1}(B, \varphi)$ is a monomorphism by Lemma 2.1. So $\sigma=\operatorname{Ext}^{n+1}(B, \varphi) \tau$ is a monomorphism. Thus, $\operatorname{Ext}^{n}(B, \beta)=0$, that is, $\beta: H \rightarrow Y$ is an $n$-Ext-phantom morphism. For any $n$-Ext-phantom morphism $\xi: G \rightarrow Y$, there exists a morphism $\psi: G \rightarrow X$ such that $g \psi=\xi$ since $g$ is an $n$-Ext-phantom precover. So $\beta(\gamma \psi)=g \psi=\xi$. Thus, $\beta: H \rightarrow Y$ is an $n$-Ext-phantom precover with $\operatorname{ker}(\beta)$ pure injective.

(2) By (1), $Y$ has an $n$-Ext-phantom precover $\beta: H \rightarrow Y$ with the kernel $C$ pure injective. Since $g$ is an $n$-Ext-phantom cover, there exists a morphism $\rho: H \rightarrow X$ such that $g \rho=\beta$. Also there exists a morphism $\omega: C \rightarrow K$ by Five Lemma such that $\lambda \omega=\rho \iota$. Then we get the following commutative diagram with exact rows:

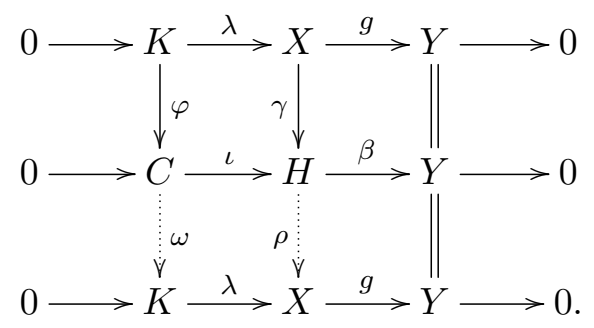

Since $g$ is an $n$-Ext-phantom cover, $\rho \gamma$ is an isomorphism. Hence, $\omega \varphi$ is an isomorphism by Five Lemma again. Thus, $K$ is isomorphic to a direct summand of $C$ and so is pure injective.

Based on the above results, we can immediately obtain the following conclusion.

Corollary 2.10. Let $R$ be a left coherent ring and $n$ a positive integer. If $\alpha: A \rightarrow B$ is an n-phantom preenvelope which is a monomorphism in Mod-R. Then, $A^{+}$has an epic $n$-Ext-phantom cover $\varphi: G \rightarrow A^{+}$in $R$-Mod such that $G$ is injective and $\operatorname{ker}(\varphi)$ is pure injective.

Proof. By Proposition 2.4, $\alpha^{+}: B^{+} \rightarrow A^{+}$is an $n$-Ext-phantom precover which is an epimorphism since $\alpha$ is a monomorphism. By [14, Corollary 2.14], $A^{+}$has an $n$-Extphantom cover $\varphi: G \rightarrow A^{+}$since $R$ is left coherent. Thus, $\varphi$ is an epimorphism since $\alpha^{+}$ is epic. By Theorem $2.9, \operatorname{ker}(\varphi)$ is pure injective. For any left $R$-module $M, M^{+}$is a pure injective right $R$-module by [5]. So $\varphi$ factors through an injective left $R$-module $E$ by [6], 
that is, there exist morphisms $\sigma: G \rightarrow E$ and $\tau: E \rightarrow A^{+}$such that $\varphi=\tau \sigma$. Since $\tau$ is an $n$-Ext-phantom morphism, there exists a morphism $\gamma: E \rightarrow G$ such that $\varphi \gamma=\tau$. So $\varphi=\varphi \gamma \sigma$. Hence, $\gamma \sigma$ is an isomorphism since $\varphi$ is an $n$-Ext-phantom cover. Thus, $G$ is isomorphic to a direct summand of $E$ and so is injective.

3. Applications of $n$-phantom envelopes and $n$-Ext-phantom covers

For a positive integer $n$, it is well known that $\mathrm{wD}(R) \leq n$ if and only if $\operatorname{Tor}_{n}^{R}(A, B)=0$ for any right $R$-module $A$ and left $R$-module $B$. Here we obtain another method to characterize the weak dimensions of a ring $R$ as follows.

Theorem 3.1. Let $R$ be a left coherent ring and $n$ a positive integer. The following statements are equivalent:

(1) $\mathrm{wD}(R) \leq n$.

(2) Every right $R$-module has an $n$-phantom envelope which is an epimorphism.

(3) Every left $R$-module has an $n$-Ext-phantom cover which is a monomorphism.

(4) Every pure injective left $R$-module has an $n$-Ext-phantom cover which is a monomorphism.

Proof. The equivalences of (1), (2) and (3) follow from [14, Theorem 2.15] and (3) $\Rightarrow(4)$ is clear. So it suffices to prove that $(4) \Rightarrow(3)$.

For any left $R$-module $X, X^{++}$has an $n$-Ext-phantom cover $\gamma: Y \rightarrow X^{++}$which is a monomorphism since $X^{++}$is pure injective. So we get the following pullback diagram

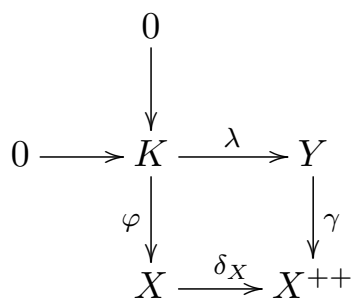

with $\lambda$ and $\varphi$ monomorphism. Since $\gamma$ is an $n$-Ext-phantom morphism, $\delta_{X} \varphi=\gamma \lambda$ is an $n$-Ext-phantom morphism. Let $F$ be a finitely presented left $R$-module, since $\delta_{X}$ is pure monomorphism and $\operatorname{Ext}^{n}\left(F, \delta_{X}\right) \operatorname{Ext}^{n}(F, \varphi)=\operatorname{Ext}^{n}(F, \gamma) \operatorname{Ext}^{n}(F, \lambda)=0, \operatorname{Ext}^{n}\left(F, \delta_{X}\right)$ is a monomorphism by Lemma 2.1 and so $\operatorname{Ext}^{n}(F, \varphi)=0$. Thus, $\varphi$ is an $n$-Ext-phantom morphism. For any $n$-Ext-phantom morphism $\psi: A \rightarrow X$, there exists a morphism $\xi: A \rightarrow$ $Y$ such that $\gamma \xi=\delta_{X} \psi$ since $\gamma$ is an $n$-Ext-phantom cover. By the universal property of a pullback diagram, there exists a morphism $\eta: A \rightarrow K$ such that $\varphi \eta=\psi$. So $\varphi$ is an $n$-Extphantom precover which is a monomorphism. It is easy to see that $\varphi$ is an $n$-Ext-phantom cover. 
In [16, Mao introduced the concepts of an $\mathcal{I}$-cover and $\mathcal{I}$-envelope with the unique mapping property, where $\mathcal{I}$ is an ideal of $\operatorname{Mod}-R$. He called $\phi: M \rightarrow F$ in $\mathcal{I}$ an $\mathcal{I}$ cover with the unique mapping property if for any morphism $f: X \rightarrow F$ in $\mathcal{I}$, there exists a unique morphism $g: X \rightarrow M$ such that $\phi g=f$. We notice that the concept of approximations with the unique mapping property are usually called reflections or coreflections in category theory since the middle of the 20th century [19, pp. 128-129]. The definition of an $\mathcal{I}$-envelope with unique mapping property is dually. Here, we have two different characterizations with regard to the weak dimensions of a ring $R$ by $n$-phantom envelope ( $n$-Ext-phantom cover) with the unique mapping property as follows.

Proposition 3.2. Let $R$ be a left coherent ring and $n$ a positive integer. If every right $R$ module has an $n$-phantom envelope with the unique mapping property, then $\mathrm{w} \mathrm{D}(R) \leq 2 n$.

Proof. For any right $R$-module $X$, we get an exact sequence

$$
0 \rightarrow K \stackrel{\psi}{\rightarrow} F_{n} \stackrel{\varphi}{\rightarrow} F_{n-1} \rightarrow \cdots \rightarrow F_{0} \rightarrow X \rightarrow 0
$$

with each $F_{i}$ flat, $i=0,1, \ldots, n$. Let $\sigma: K \rightarrow H$ be an $n$-phantom envelope of $K$ with the unique mapping property. Since $\psi$ is an $n$-phantom morphism, there exists a morphism $\tau: H \rightarrow F_{n}$ such that $\tau \sigma=\psi$. Then $\varphi \tau \sigma=\varphi \psi=0$. Clearly, $0 \sigma=0$. We note that $\sigma$ is an $n$-phantom envelope with the unique mapping property. Thus, $\varphi \tau=0$. By the property of exact sequence in the category of modules, there exists a morphism $\alpha: H \rightarrow K$ such that $\psi \alpha=\tau$. Since $\psi \alpha \sigma=\tau \sigma=\psi$ and $\psi$ is a monomorphism, we have $\alpha \sigma=1_{K}$. This implies that $\sigma$ is an $n$-phantom and pure monomorphism. Thus, by Proposition $2.2(4)$, we have $\mathrm{fd}(K) \leq n-1$ and $\operatorname{so} \mathrm{fd}(X) \leq 2 n$. Therefore, $\mathrm{wD}(R) \leq 2 n$.

Now, for this consequence above, if we make the condition "every right $R$-module has an $n$-phantom envelope" into "every left $R$-module has an $n$-Ext-phantom cover", then we can use another method to prove the above theorem as follows.

Proposition 3.3. Let $R$ be a left coherent ring and $n$ a positive integer. If every left $R$ module has an $n$-Ext-phantom cover with the unique mapping property, then $\mathrm{wD}(R) \leq 2 n$.

Proof. For any left $R$-module $Y$, we get an exact sequence

$$
0 \rightarrow Y \rightarrow E^{0} \rightarrow \cdots \rightarrow E^{n-1} \stackrel{\alpha}{\rightarrow} E^{n} \stackrel{\beta}{\rightarrow} C \rightarrow 0
$$

with each $E^{i}$ injective, $i=0,1, \ldots, n$. Let $\sigma: H \rightarrow C$ be an $n$-Ext-phantom cover with the unique mapping property. Since $\beta$ is an $n$-Ext-phantom morphism, there exists a morphism $\eta: E^{n} \rightarrow H$ such that $\sigma \eta=\beta$. Then $\sigma \eta \alpha=\beta \alpha=0$. Clearly, $\sigma 0=0$. We note that $\sigma$ is an $n$-Ext-phantom cover with the unique mapping property. Thus, $\eta \alpha=0$. By the property of exact sequence in the category of modules, there exists a morphism 
$\gamma: C \rightarrow H$ such that $\gamma \beta=\eta$ and so $\sigma \gamma \beta=\sigma \eta=\beta$. Since $\beta$ is an epimorphism, we have $\sigma \gamma=1_{C}$. This implies that $\sigma$ is an $n$-Ext-phantom and pure epimorphism. Then, by Proposition $2.3(4)$, we have $\mathrm{FP}-\mathrm{id}(C) \leq n-1$ and so $\mathrm{FP}-\mathrm{id}(Y) \leq 2 n$. Therefore, $\mathrm{wD}(R) \leq 2 n$.

By [15, Definition 2.1], a morphism $f: M \rightarrow N$ of left $R$-modules is called Tor $_{n^{-}}$ epimorphism if the induced morphism

$$
\operatorname{Tor}_{n}(A, f): \operatorname{Tor}_{n}(A, M) \rightarrow \operatorname{Tor}_{n}(A, N)
$$

of abelian groups is an epimorphism for every (finitely presented) right $R$-module $A$. And a morphism $g: X \rightarrow Y$ of left $R$-modules is called an Ext ${ }^{n}$-monomorphism if the induced morphism

$$
\operatorname{Ext}^{n}(B, g): \operatorname{Ext}^{n}(B, X) \rightarrow \operatorname{Ext}^{n}(B, Y)
$$

of abelian groups is a monomorphism for every finitely presented left $R$-module $B$.

Dually, we call a morphism $f: M \rightarrow N$ of left $R$-modules Ext $^{n}$-epimorphism if the induced morphism

$$
\operatorname{Ext}^{n}(A, f): \operatorname{Ext}^{n}(A, M) \rightarrow \operatorname{Ext}^{n}(A, N)
$$

of abelian groups is an epimorphism for every finitely presented left $R$-module $A$. And a morphism $g: X \rightarrow Y$ of left $R$-modules is called a Tor $_{n}$-monomorphism if the induced morphism

$$
\operatorname{Tor}_{n}(B, g): \operatorname{Tor}_{n}(B, X) \rightarrow \operatorname{Tor}_{n}(B, Y)
$$

of abelian groups is a monomorphism for every (finitely presented) right $R$-module $B$.

In the end of this paper, we give some characterizations on the relationship between $n$ Ext-phantom morphisms and Ext ${ }^{n}$-monomorphisms and Ext ${ }^{n}$-epimorphisms and the relationship between $n$-phantom morphisms and Tor $_{n}$-epimorphisms and Tor $_{n}$-monomorphisms as follows.

Proposition 3.4. Let $R$ be a ring, $n$ a positive integer and $\left(A_{1} \stackrel{\tau}{\rightarrow} A_{2}\right) \stackrel{\left(f_{1}, g_{1}\right)}{\longrightarrow}\left(B_{1} \stackrel{\sigma}{\rightarrow}\right.$ $\left.B_{2}\right) \stackrel{\left(f_{2}, g_{2}\right)}{\longrightarrow}\left(C_{1} \stackrel{\gamma}{\rightarrow} C_{2}\right)$ a composition of two morphisms in $R$-Mor. If $g_{1}$ is an $\mathrm{Ext}^{n}$ monomorphism, $f_{2}$ is an Ext $^{n}$-epimorphism and $\sigma$ an $n$-Ext-phantom morphism. Then both $\tau$ and $\gamma$ are $n$-Ext-phantom morphisms.

Proof. Let $X$ be any finitely presented left $R$-module. By the conditions, we get the following commutative diagram with exact rows:

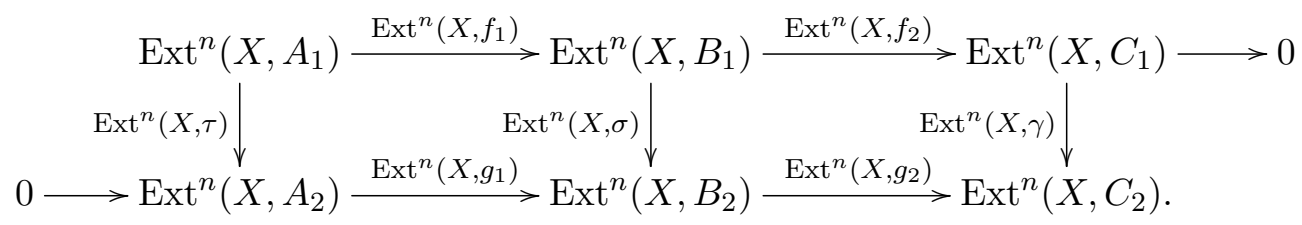


Since $\sigma$ is an $n$-Ext-phantom morphism, we have

$$
\operatorname{Ext}^{n}\left(X, \sigma f_{1}\right)=\operatorname{Ext}^{n}(X, \sigma) \operatorname{Ext}^{n}\left(X, f_{1}\right)=0
$$

So

$$
\operatorname{Ext}^{n}\left(X, g_{1} \tau\right)=\operatorname{Ext}^{n}\left(X, g_{1}\right) \operatorname{Ext}^{n}(X, \tau)=\operatorname{Ext}^{n}\left(X, \sigma f_{1}\right)=0 .
$$

Since $g_{1}$ is an $\operatorname{Ext}^{n}$-monomorphism, $\operatorname{Ext}^{n}(X, \tau)=0$. Thus, $\tau$ is an $n$-Ext-phantom morphism. On the other hand, by the commutativity of the right square of the diagram above, we have

$$
\operatorname{Ext}^{n}\left(X, g_{2} \sigma\right)=\operatorname{Ext}^{n}\left(X, g_{2}\right) \operatorname{Ext}^{n}(X, \sigma)=0
$$

So

$$
\operatorname{Ext}^{n}\left(X, \gamma f_{2}\right)=\operatorname{Ext}^{n}(X, \gamma) \operatorname{Ext}^{n}\left(X, f_{2}\right)=\operatorname{Ext}^{n}\left(X, g_{2} \sigma\right)=0
$$

Since $f_{2}$ is an $\operatorname{Ext}^{n}$-epimorphism, $\operatorname{Ext}^{n}(X, \gamma)=0$. Thus, $\gamma$ is also an $n$-Ext-phantom morphism.

Proposition 3.5. Let $R$ be a ring, $n$ a positive integer and $\left(X_{1} \stackrel{\alpha}{\rightarrow} X_{2}\right) \stackrel{\left(h_{1}, k_{1}\right)}{\longrightarrow}\left(Y_{1} \stackrel{\beta}{\rightarrow}\right.$ $\left.Y_{2}\right) \stackrel{\left(h_{2}, k_{2}\right)}{\longrightarrow}\left(Z_{1} \stackrel{\theta}{\rightarrow} Z_{2}\right)$ a composition of two morphisms in $R$-Mor. If $h_{2}$ is a Tor $_{n-}$ epimorphism, $k_{1}$ is a Tor $_{n}$-monomorphism and $\beta$ is an n-phantom morphism. Then both $\alpha$ and $\theta$ are $n$-phantom morphisms.

Proof. Let $M$ be any right $R$ module. By the conditions, we get the following commutative diagram with exact rows:

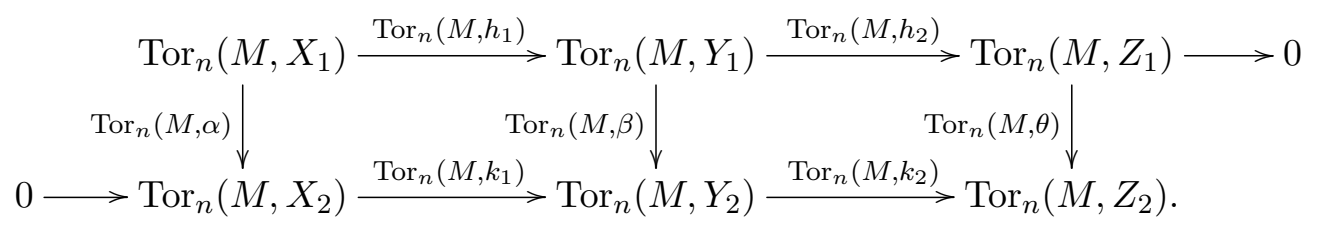

Since $\beta$ is an $n$-phantom morphism, we have

$$
\operatorname{Tor}_{n}\left(M, k_{2} \beta\right)=\operatorname{Tor}_{n}\left(M, k_{2}\right) \operatorname{Tor}_{n}(M, \beta)=0 .
$$

So

$$
\operatorname{Tor}_{n}\left(M, \theta h_{2}\right)=\operatorname{Tor}_{n}(M, \theta) \operatorname{Tor}_{n}\left(M, h_{2}\right)=\operatorname{Tor}_{n}\left(M, k_{2} \beta\right)=0 .
$$

Since $h_{2}$ is $\operatorname{Tor}_{n}$-epimorphism, $\operatorname{Tor}_{n}(M, \theta)=0$. Therefore, $\beta$ is an $n$-phantom morphism. On the other hand, by the commutativity of the left square of the diagram above, we have

$$
\operatorname{Tor}_{n}\left(M, \beta h_{1}\right)=\operatorname{Tor}_{n}(M, \beta) \operatorname{Tor}_{n}\left(M, h_{1}\right)=0 .
$$

So

$$
\operatorname{Tor}_{n}\left(M, k_{1} \alpha\right)=\operatorname{Tor}_{n}\left(M, k_{1}\right) \operatorname{Tor}_{n}(M, \alpha)=\operatorname{Tor}_{n}\left(M, \beta h_{1}\right)=0
$$

Since $k_{1}$ is a Tor $_{n}$-monomorphism, $\alpha$ is an $n$-phantom morphism. 
Proposition 3.6. Let $R$ be a ring and $n$ a positive integer. For two exact sequences of left $R$-modules $0 \rightarrow A \stackrel{\alpha}{\rightarrow} B \stackrel{\beta}{\rightarrow} E \rightarrow 0$ and $0 \rightarrow A \stackrel{\sigma}{\rightarrow} C \stackrel{\tau}{\rightarrow} F \rightarrow 0$ with $\tau: C \rightarrow F$ an Ext $^{n}$-epimorphism. Consider the following pushout diagram

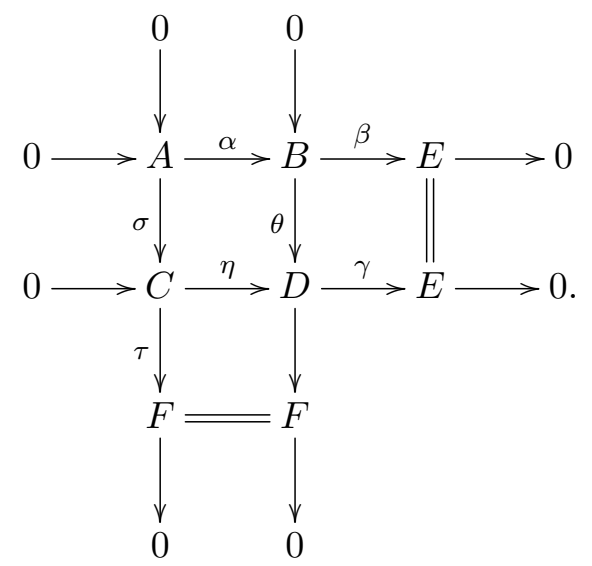

Then

(1) $\beta$ is an $n$-Ext-phantom morphism if and only if $\gamma$ is an $n$-Ext-phantom morphism.

(2) $\beta$ is an $\mathrm{Ext}^{n}$-epimorphism if and only if $\gamma$ is an $\mathrm{Ext}^{n}$-epimorphism.

Proof. Let $X$ be any finitely presented left $R$-module. By the exact sequence $0 \rightarrow A \stackrel{\sigma}{\rightarrow}$ $C \stackrel{\tau}{\rightarrow} F \rightarrow 0$, we get the exact sequence

$$
\operatorname{Ext}^{n}(X, C) \stackrel{\operatorname{Ext}^{n}(X, \tau)}{\longrightarrow} \operatorname{Ext}^{n}(X, F) \longrightarrow \operatorname{Ext}^{n+1}(X, A) \stackrel{\operatorname{Ext}^{n+1}(X, \sigma)}{\longrightarrow} \operatorname{Ext}^{n+1}(X, C) .
$$

Since $\operatorname{Ext}^{n}(X, \tau)$ is an epimorphism, $\operatorname{Ext}^{n+1}(X, \sigma)$ is a monomorphism.

Let us consider the following commutative diagram with exact rows:

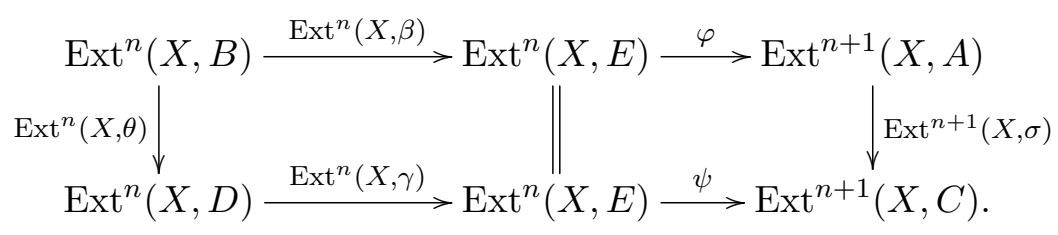

Then, (1) $\beta$ is an $n$-Ext-phantom morphism if and only if $\varphi$ is a monomorphism if and only if $\psi$ is a monomorphism if and only if $\gamma$ is an $n$-Ext-phantom morphism.

(2) $\beta$ is an Ext $^{n}$-epimorphism if and only if $\varphi=0$ if and only if $\psi=0$ if and only if $\gamma$ is an $\mathrm{Ext}^{n}$-epimorphism and so we finished the whole works.

\section{Acknowledgments}

This work was supported by National Natural Science Foundation of China (No. 12061061), Fundamental Research Funds for the Central Universities (No. 31920190057) and Innovation Team Project of Northwest Minzu University (No. 1110130131). 


\section{References}

[1] D. J. Benson, Phantom maps and purity in modular representation theory III, J. Algebra 248 (2002), no. 2, 747-754.

[2] R. F. Damiano, Coflat rings and modules, Pacific J. Math. 81 (1979), no. 2, 349-369.

[3] N. Q. Ding and J. L. Chen, Relative coherence and preenvelopes, Manuscripta Math. 81 (1993), no. 3-4, 243-262.

[4] E. E. Enochs, Injective and flat covers, envelopes and resolvents, Israel J. Math. 39 (1981), no. 3, 189-209.

[5] E. E. Enochs and O. M. G. Jenda, Relative Homological Algebra, De Gruyter Expositions in Mathematics 30, Walter de Gruyter, Berlin, 2000.

[6] X. H. Fu, P. A. Guil Asensio, I. Herzog and B. Torrecillas, Ideal approximation theory, Adv. Math. 244 (2013), 750-790.

[7] X. H. Fu and I. Herzog, Powers of the phantom ideal, Proc. Lond. Math. Soc. (3) 112 (2016), no. 4, 714-752.

[8] I. Herzog, The phantom cover of a module, Adv. Math. 215 (2007), no. 1, 220-249.

[9] _ Contravariant functors on the category of finitely presented modules, Israel J. Math. 167 (2008), 347-410.

[10] S. Jain, Flat and FP-injectivity, Proc. Amer. Math. Soc. 41 (1973), 437-442.

[11] T. Y. Lam, Lectures on Modules and Rings, Graduate Texts in Mathematics 189, Springer-Verlag, New York, 1999.

[12] Q. Liu and J. Chen, Coherence and generalized morphic property of triangular matrix rings, Comm. Algebra 42 (2014), no. 7, 2788-2799.

[13] L. Mao, On covers and envelopes in some functor categories, Comm. Algebra 41 (2013), no. 5, 1655-1684.

[14] _ Higher phantom and Ext-phantom morphisms, J. Algebra Appl. 17 (2018), no. 1, 1850012, 15 pp.

[15] _ Higher phantom morphisms with respect to a subfunctor of Ext, Algebr. Represent. Theory 22 (2019), no. 2, 407-424. 
[16] _ Phantom envelopes and Ext-phantom covers of modules, Bull. Iranian Math. Soc. 46 (2020), no. 2, 441-455.

[17] L. Mao and N. Ding, Envelopes and covers by modules of finite FP-injective and flat dimensions, Comm. Algebra 35 (2007), no. 3, 833-849.

[18] C. A. McGibbon, Phantom maps, in: Handboook of Algebraic Topology, 1209-1257, North-Holland, Amsterdam, 1995.

[19] B. Mitchell, Theory of Categories, Pure and Applied Mathematics XVII, Academic Press, New York, 1965.

[20] A. Neeman, The Brown representability theorem and phantomless triangulated categories, J. Algebra 151 (1992), no. 1, 118-155.

[21] J. J. Rotman, An Introduction to Homological Algebra, Pure and Applied Mathematics 85, Academic Press, New York, 1979.

[22] B. Stenström, Coherent rings and FP-injective modules, J. London Math. Soc. (2) 2 (1970), 323-329.

[23] R. B. Warfield Jr., Purity and algebraic compactness for modules, Pacific J. Math. 28 (1969), no. 3, 699-719.

Kaiyang Lan and Bo Lu

College of Mathematics and Computer Science, Northwest Minzu University, Lanzhou 730030, China

E-mail addresses: 745365423@qq.com, lubo55@126.com 